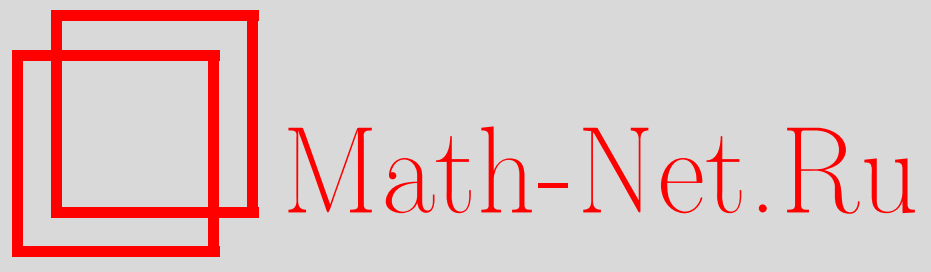

В. Л. Верещагин, Солитонные решения интегрируемой граничной задачи на полуоси для дискретной цепочки Тоды, ТМФ, 2006, том 148, номер 3, 387-397

DOI: https://doi.org/10.4213/tmf2323

Использование Общероссийского математического портала Math-Net.Ru подразумевает, что вы прочитали и согласны с пользовательским соглашением http://www . mathnet.ru/rus/agreement

Параметры загрузки:

IP: 34.229 .108 .108

26 апреля 2023 г., 14:06:11

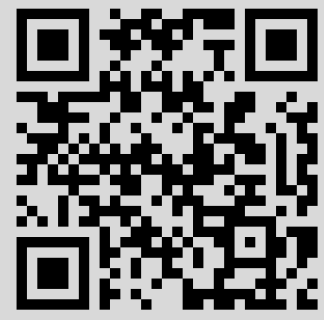




\title{
СОЛИТОННЫЕ РЕШЕНИЯ ИНТЕГРИРУЕМОЙ ГРАНИЧНОЙ ЗАДАЧИ НА ПОЛУОСИ ДЛЯ ДИСКРЕТНОЙ ЦЕПОЧКИ ТОДЫ
}

\begin{abstract}
Выписаны формулы для солитонных решений дискретной цепочки Тоды и поставлена интегрируемая граничная задача для этой цепочки. Найдены условия для параметров (точек дискретного спектра, коэффициентов прохождения и соответствующих множителей), выделяющие среди всех солитонных решений те, которые отвечают решениям интегрируемой граничной задачи. В результате построены две иерархии солитонных решений с четными и нечетными номерами указанной задачи, причем условия для параметров представлены в явном виде.
\end{abstract}

Ключевые слова: дискретная цепочка Тоды, интегрируемая краевая задача, солитон.

\section{1. ВВЕДЕНИЕ}

В последние десятилетия значительно вырос интерес к интегрируемым нелинейным уравнениям для величин, зависящих только от дискретных (целых) переменных. В работе [1] был приведен ряд важных примеров таких систем, в число которых вошел главный объект рассмотрения данной статьи - так называемая дискретная цепочка Тоды

$$
q_{m+1, n}-2 q_{m, n}+q_{m-1, n}=\ln \frac{e^{q_{m, n+1}-q_{m, n}}+1}{e^{q_{m, n}-q_{m, n-1}}+1}
$$

или, в обозначениях $v_{m, n}=e^{q_{m, n}}$,

$$
v_{m-1, n}\left(\frac{1}{v_{m, n}}+\frac{1}{v_{m, n-1}}\right)=\frac{1}{v_{m+1, n}}\left(v_{m, n+1}+v_{m, n}\right),
$$

где $q_{m, n}, m, n \in \mathbb{Z},-$ неизвестная функция двух дискретных аргументов. Была показана полная интегрируемость системы (1.1) в смысле наличия матричного представления нулевой кривизны

$$
L_{m+1, n}(\lambda) A_{m, n}(\lambda)=A_{m, n+1}(\lambda) L_{m, n}(\lambda),
$$

*Институт математики с ВЦ УНЦ РАН, Уфа, Россия. E-mail: v_vereschagin@mail.ru 
где

$$
\begin{aligned}
L_{m, n}(\lambda) & =\left(\begin{array}{cc}
\lambda+e^{q_{m, n}-q_{m-1, n}} & e^{q_{m, n}} \\
\lambda e^{-q_{m-1, n}} & 0
\end{array}\right), \\
A_{m, n}(\lambda) & =\left(\begin{array}{cc}
\lambda & e^{q_{m, n}} \\
\lambda e^{-q_{m, n-1}} & -1
\end{array}\right)
\end{aligned}
$$

$\lambda$ - комплексный спектральный параметр. Следствием полной интегрируемости является возможность явного решения задачи Коши (по аргументу $m$ ) в случае быстроубывающего или квазипериодического (по $n$ ) начального условия.

Сравнительно более новым и актуальным стал вопрос о корректной постановке интегрируемой граничной задачи для дискретной цепочки Тоды. Дело в том, что при произвольном выборе граничного (при фиксированном $n$ ) условия на $q_{m, n}$ система (1.1) теряет свойство интегрируемости, т.е. наличия полного набора законов сохранения. Поэтому актуальна проблема выбора таких граничных условий, которые позволяют сохранить свойство интегрируемости. Впервые ряд частных примеров был приведен в работе [2]. Оказалось, что при наложении таких (назовем их интегрируемыми) граничных условий остаются хотя и не все, но достаточно многие законы сохранения, необходимые для формирования полного набора атрибутов интегрируемости. Позже в работах [3]-[5] была построена соответствующая теория интегрируемых граничных условий и выписан набор примеров, обобщающих примеры, полученные в работе [2]. В частности, для уравнения (1.1) граничное условие в точке $n=0$ имеет вид

$$
\frac{1}{v_{m, 0}}=\frac{\hat{a} v_{m, 1}}{c^{4 m}}+\frac{\hat{b}}{c^{2 m}},
$$

где $\hat{a}, \hat{b}$ - константы, $v_{m, n}=e^{q_{m, n}}$, а параметр $c$ в дальнейшем будем считать равным единице. Такое граничное условие называется совместимым с уравнением нулевой кривизны (1.2), т.е. линейное уравнение

$$
Y_{m+1,0}(\lambda)=A_{m, 0}(\lambda) Y_{m, 0}(\lambda)
$$

для $(2 \times 2)$-матрицы $Y$ при выполнении условия (1.4) обладает дополнительной точечной симметрией вида

$$
\widetilde{Y}_{m, 0}(\tilde{\lambda})=H_{m}(q, \lambda) Y_{m, 0}(\lambda), \quad \tilde{\lambda}=\lambda^{-1} .
$$

Существование матрицы $H_{m}(q, \lambda)$ и, таким образом, инволюции $\tilde{\lambda}(\lambda)$ является признаком интегрируемости граничного условия и обеспечивает интегрируемость потока по переменной $m$ после наложения условия (1.4).

Тем не менее остается открытым вопрос о конкретном виде решений упомянутых интегрируемых граничных задач для системы (1.1). Основной целью данной статьи является частичное заполнение этого пробела, а именно:

а) выписывание солитонных решений дискретной цепочки Тоды (1.1);

б) выделение в явном виде среди этих решений тех, которые решают интегрируемую граничную задачу.

Эти цели определяют структуру работы: в разделе 2 выписывается набор солитонных решений дискретной цепочки Тоды, в разделе 3 определяются условия, 
налагаемые на дискретный спектр наличием граничного условия, в разделе 4 описываются остальные параметры солитонных решений, в разделе 5 предъявлена процедура построения явных формул для солитонных решений интегрируемой граничной задачи.

\section{2. СОЛИТОННЫЕ РЕШЕНИЯ ДИСКРЕТНОЙ ЦЕПОЧКИ ТОДЫ}

Метод обратной задачи рассеяния (см., например, книгу [6]), используемый здесь для отыскания солитонов, является стандартным и восходит к решению соответствующей задачи Римана.

Для удобства перепишем матрицы $L, A$ в следующем виде:

$$
\begin{aligned}
& L_{m, n}(\lambda)=\left(\begin{array}{cc}
-\lambda+\frac{1}{\lambda} \frac{v_{m, n}}{v_{m-1, n}} & \frac{v_{m, n}}{\lambda} \\
-\frac{\lambda}{v_{m-1, n}} & 0
\end{array}\right), \\
& A_{m, n}(\lambda)=\frac{1}{\lambda \sqrt{2}}\left(\begin{array}{cc}
-\lambda^{2} & v_{m, n} \\
-\frac{\lambda^{2}}{v_{m, n-1}} & -1
\end{array}\right),
\end{aligned}
$$

где $v_{m, n}=e^{q_{m, n}}$. Если $(2 \times 2)$-матрица $Y_{m, n}-$ решение линейного уравнения

$$
Y_{m, n+1}(\lambda)=L_{m, n}(\lambda) Y_{m, n}(\lambda),
$$

то, обозначив $\Psi_{m, n}(\lambda)=Y_{m, n}^{11}(\lambda)$, получим уравнение второго порядка для скалярной функции $\Psi_{m, n}(\lambda)$ :

$$
\psi_{m, n+1}(\lambda)=\left(-\lambda+\frac{v_{m, n}}{\lambda v_{m-1, n}}\right) \psi_{m, n}(\lambda)-\frac{v_{m, n}}{v_{m-1, n-1}} \psi_{m, n-1}(\lambda) .
$$

Будем искать солитонные, т.е. быстро стремящиеся к константе при $n \rightarrow \pm \infty$, решения $q_{m, n}$ системы (1.1). Следовательно, не теряя общности, при $n \rightarrow \pm \infty$ имеем оценку

$$
L_{m, n}(\lambda) \rightarrow U\left(\begin{array}{cc}
z & 0 \\
0 & z^{-1}
\end{array}\right) U^{-1}
$$

где $U$ - некоторая матрица, параметры $\lambda$ и $z$ связаны соотношением

$$
\lambda^{-1}-\lambda=z^{-1}+z \text {. }
$$

Имеем $\operatorname{det} U=\mathrm{const} \cdot\left(z^{2}-1\right)$, следовательно, непрерывный спектр линейной задачи (2.2) имеет вид единичной окружности $|z|=1$, а значения $z= \pm 1$ являются виртуальными уровнями.

Таким образом, можно определить две пары базисных решений уравнения (2.3) с помощью асимптотик при $n \rightarrow \pm \infty$ (переменную $m$ пока считаем произвольной):

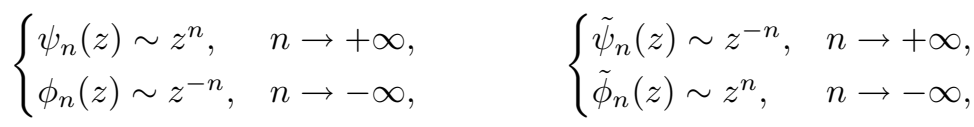

причем функции $\psi_{n}(z), \phi_{n}(z)$ аналитичны внутри круга с границей $|z|=1$, а функции $\tilde{\psi}_{n}(z), \tilde{\phi}_{n}(z)$ - вне его. Свяжем эти решения следующим образом:

$$
\psi_{n}(z)=a(z) \tilde{\phi}_{n}(z)+b(z) \phi_{n}(z)
$$


где $a(z), b(z)$ зависят только от параметра $z$. На окружности $|z|=1$ имеем

$$
\tilde{\phi}_{n}(z)=\bar{\phi}_{n}(z), \quad \tilde{\psi}_{n}(z)=\bar{\psi}_{n}(z), \quad|z|=1,
$$

где черта сверху означает комплексное сопряжение. Отсюда, в частности, вытекают следующие свойства величин $a(z), b(z)$ :

$$
|a(z)|^{2}-|b(z)|^{2}=1, \quad a(-z)=a(z), \quad b(-z)=b(z), \quad|z|=1 .
$$

Продолжим равенства $(2.8)$ с окружности $|z|=1$ на комплексную плоскость $\mathbb{C}$ :

$$
\tilde{\phi}_{n}(z)=\phi_{n}\left(z^{-1}\right), \quad \tilde{\psi}_{n}(z)=\psi_{n}\left(z^{-1}\right), \quad z \in \mathbb{C} .
$$

Теперь введем обозначения

$$
\psi_{n}(z)=z^{n} \theta_{n}(z), \quad \tilde{\phi}_{n}(z)=z^{n} \tilde{\chi}_{n}(z), \quad \phi_{n}(z)=z^{-n} \chi_{n}(z),
$$

откуда в силу (2.7) получаем

$$
\frac{\theta_{n}(z)}{a(z)}=\tilde{\chi}_{n}(z)+r(z) \chi_{n}(z), \quad|z|=1,
$$

где $r(z)=b(z) / a(z)$. Сформулируем следующую задачу Римана с контуром в виде единичной окружности $|z|=1$ : построить кусочно-аналитическую функцию $\Phi_{n}(z)$ такую, что

$$
\Phi_{n}(z)= \begin{cases}\frac{\theta_{n}(z)}{a(z)}, & |z|<1 \\ \tilde{\chi}_{n}(z), & |z|>1 .\end{cases}
$$

Функция $\Phi_{n}(z)$ имеет простые полюсы внутри круга $|z|<1$ в нулях коэффициента $a(z): \quad a\left(z_{k}\right)=0, k=1,2, \ldots, N$, с вычетами

$$
\operatorname{Res}_{z=z_{k}} \Phi_{n}(z)=\operatorname{Res}_{z=z_{k}} \frac{\theta_{n}(z)}{\left(z-z_{k}\right) a^{\prime}\left(z_{k}\right)}=\frac{b_{k} z_{k}^{-2 n} \tilde{\chi}_{n}\left(z_{k}^{-1}\right)}{a^{\prime}\left(z_{k}\right)}
$$

в силу $\tilde{\chi}_{n}\left(z^{-1}\right)=\chi_{n}(z)$. Величины $z_{k}$ являются точками дискретного спектра. На окружности $|z|=1$ функция $\Phi_{n}(z)$ испытывает скачок, равный $z^{-2 n} r(z) \chi_{n}(z)$. Нормируем $\Phi_{n}(z)$ при $|z| \rightarrow \infty$ условием $\Phi_{n}(z) \rightarrow 1$ и получаем решение задачи Римана $(2.12)$ :

$$
\Phi_{n}(z)=1+\sum_{k=1}^{N} \frac{b_{k} z_{k}^{-2 n} \tilde{\chi}_{n}\left(z_{k}^{-1}\right)}{a^{\prime}\left(z_{k}\right)}-\frac{1}{2 \pi i} \int_{|\xi|=1} \frac{\xi^{-2 n} r(\xi) \chi_{n}(\xi) d \xi}{\xi-z}
$$

где $b_{k}-$ коэффициент, связывающий $\psi_{n}, \phi_{n}$ в точке $z_{k}: \psi_{n}\left(z_{k}\right)=b_{k} \phi_{n}\left(z_{k}\right)$. Положив в формуле (2.13) $z=0$, получаем

$$
\begin{gathered}
\Phi_{n}(0)=\frac{\theta_{n}(0)}{a(0)}=1-\sum_{m=1}^{N} z_{m}^{-2 n-1} b_{m}\left(a^{\prime}\left(z_{m}\right)\right)^{-1} \Gamma_{n}^{(m)}- \\
-\frac{1}{2 \pi i} \int_{|\xi|=1} \xi^{-2 n-1} r(\xi) \chi_{n}(\xi) d \xi
\end{gathered}
$$


где $\Gamma_{n}^{(m)}=\tilde{\chi}_{n}\left(z_{m}^{-1}\right)$. С другой стороны, при $|z|>1 \quad \Phi_{n}(z)=\tilde{\chi}_{n}(z)$, и для $z=z_{k}^{-1}$ соотношение (2.13) дает

$$
\begin{aligned}
\Gamma_{n}^{(k)}=1 & +\sum_{m=1}^{N} z_{m}^{-2 n} b_{m}\left(a^{\prime}\left(z_{m}\right)\right)^{-1} \frac{\Gamma_{n}^{(m)}}{z_{k}^{-1}-z_{m}}- \\
& -\frac{1}{2 \pi i} \int_{|\xi|=1} \frac{\xi^{-2 n} r(\xi) \chi_{n}(\xi) d \xi}{\xi-z_{k}^{-1}}, \quad k=1,2, \ldots, N .
\end{aligned}
$$

Примем коэффициент отражения равным нулю: $r(z) \equiv 0$, т.е. будем рассматривать так называемый "безотражательный” случай. Тогда система (2.15) становится замкнутой относительно величин $\Gamma_{n}^{(k)}, k=1,2, \ldots, N$ :

$$
\Gamma_{n}^{(k)}=1+\sum_{m=1}^{N} z_{m}^{-2 n} b_{m}\left(a^{\prime}\left(z_{m}\right)\right)^{-1} \frac{\Gamma_{n}^{(m)}}{z_{k}^{-1}-z_{m}}, \quad k=1,2, \ldots, N .
$$

Решив систему (2.16), т.е. выразив $\Gamma_{n}^{(k)}$ через набор данных рассеяния $\left(z_{k}, b_{k} / a^{\prime}\left(z_{k}\right)\right.$, $k=1,2, \ldots, N)$, из $(2.14)$ получим

$$
\frac{\theta_{n}(0)}{a(0)}=1-\sum_{m=1}^{N} z_{m}^{-2 n-1} b_{m}\left(a^{\prime}\left(z_{m}\right)\right)^{-1} \Gamma_{n}^{(m)} .
$$

С другой стороны, рассматривая линейное уравнение (2.3) в окрестности точки $z=0$, получим формулу для солитонного решения:

$$
v_{m, n}=\operatorname{const} \cdot \theta_{m, n}(0)=\hat{c}\left(1-\sum_{j=1}^{N} z_{j}^{-2 n-1} \beta_{j}^{m} \Gamma_{n}^{(j)}\right) .
$$

Осталось лишь найти динамику данных рассеяния по переменной $m$. Используем исходную линейную задачу (см. (2.1)):

$$
\left.\begin{array}{c}
\beta_{j}^{m}=b_{j}^{m}\left(a^{\prime}\left(z_{j}\right)\right)^{-1}, \\
\phi_{m, n}(z) \\
Y_{m, n}^{21}(z)
\end{array}\right) \rightarrow \begin{cases}\tilde{c}_{m}\left(\begin{array}{c}
z^{-n} \\
-\lambda z^{-n+1}
\end{array}\right), & n \rightarrow-\infty \\
\tilde{c}_{m}\left(\begin{array}{c}
a_{m} z^{-n}+b_{m} z^{n} \\
-\lambda\left(a_{m} z^{-n+1}+b_{m} z^{n-1}\right)
\end{array}\right), & n \rightarrow+\infty .\end{cases}
$$

Замыкая систему при $n \rightarrow-\infty$, получаем $\tilde{c}_{m}=(\lambda / z-1)^{m}$ и принимая во внимание, что

$$
Y_{m+1, n} \rightarrow \frac{1}{\lambda \sqrt{2}}\left(\begin{array}{cc}
-\lambda^{2} & 1 \\
-\lambda^{2} & -1
\end{array}\right) Y_{m, n}
$$

при $n \rightarrow+\infty$, выводим

$$
a_{m}(z) \equiv a_{0}(z), \quad b_{m}(z)=b_{0}(z)\left[\frac{\lambda(1+z \lambda)}{z-\lambda}\right]^{m}, \quad m \in \mathbb{Z} .
$$


Аналогичным образом выводится зависимость коэффициентов $b_{k}^{m}$ от переменной $m$ :

$$
b_{k}^{m}=\left(\xi_{k}\right)^{2 m} b_{k}^{0}, \quad \xi_{k}=\frac{z_{k}-\lambda_{k}^{-1}}{\sqrt{2}} .
$$

Используя эти соотношения, найдем значение $\beta_{j}^{m}$, подставим его и получим солитонные решения дискретной цепочки Тоды. Простейшее односолитонное решение имеет вид

при условии, что

$$
e^{q_{m, n}}=v_{m, n}=\hat{c} \frac{1+\beta_{1}\left(\xi_{1}\right)^{2 m}\left(z_{1}\right)^{-2(n+1)}}{1+\beta_{1}\left(\xi_{1}\right)^{2 m}\left(z_{1}\right)^{-2 n}}
$$

$$
c_{1}=-\frac{b_{0}^{1}}{a^{\prime}\left(z_{1}\right)\left(z_{1}^{-1}-z_{1}\right)}, \quad \xi_{1}=\frac{1}{2 \sqrt{2}}\left(z_{1}-z_{1}^{-1} \pm \sqrt{z_{1}^{2}+z_{1}^{-2}+6}\right),
$$

$\hat{c}$ - произвольная константа.

\section{3. РЕШЕНИЯ ГРАНИЧНОЙ ЗАДАЧИ}

В работе [4] было представлено следующее граничное условие для системы (1.1):

$$
\frac{1}{v_{m, 0}}=\hat{a} v_{m, 1}+\hat{b}
$$

где $\hat{a}, \hat{b}$ - произвольные постоянные, и было показано, что оно является интегрируемым с инволюцией $\tilde{\lambda}=\lambda^{-1}$. В данном разделе мы будем строить солитонные решения граничной задачи (1.1), (3.1). Прежде всего отметим, что условие (3.1) задает постоянные значения, к которым может стремиться солитонное решение при $m \rightarrow \pm \infty:$

$$
v \rightarrow \hat{c}^{ \pm}=\frac{-\hat{b} \pm \sqrt{\hat{b}^{2}+4 \hat{a}}}{2 \hat{a}} .
$$

Следуя определению интегрируемости граничного условия, отметим существование матричной функции $H_{m}(q, \lambda)$ такой, что

$$
H_{m+1}(q, \lambda) A_{m, 0}(\lambda)=A_{m, 0}(\tilde{\lambda}) H_{m}(q, \lambda) .
$$

Требуется среди всех решений $Y(\lambda)$ линейной задачи

$$
Y_{m+1, n}(\lambda)=A_{m, n}(\lambda) Y_{m, n}(\lambda)
$$

выделить те, которые при $n=0$ удовлетворяют условию наличия дополнительной точечной симметрии:

$$
\tilde{Y}_{m, 0}(\tilde{\lambda})=H_{m}(q, \lambda) Y_{m, 0}(\lambda),
$$

т.е. функция $\widetilde{Y}$ снова должна быть решением уравнения (3.3) при $n=0$.

Подберем калибровочное преобразование

$$
A_{m}(\lambda) \rightarrow \hat{A}_{m}(\lambda)=F_{m+1}(\lambda) A_{m}(\lambda) F_{m}^{-1}(\lambda),
$$

соответствующее условию $(3.4)$, так, что матрица $\hat{A}_{m}(\lambda)$ "не чувствует" инволюции $\tilde{\lambda}$ :

$$
\hat{A}_{m}(\tilde{\lambda})=\hat{A}_{m}(\lambda), \quad \lambda \in \mathbb{C} .
$$


Лемма 1. Матрица $F_{m}(\lambda)$, задающая калибровочное преобразование (3.5) при условии (3.6), имеет вид

$$
F_{m}(\lambda)=\frac{1}{\sqrt{\lambda}}\left(\begin{array}{cc}
\sqrt{-\hat{a}} \lambda & 1 \\
\sqrt{-\hat{a}} \lambda \eta & \eta
\end{array}\right)
$$

где $\eta=\left(\lambda^{-1}-\lambda\right) \sqrt{-\hat{a}}-\hat{b}$.

ДокАЗАТЕльство. Представим матрицу $\hat{A}_{m}(\lambda)$ в следующем виде:

$$
\begin{aligned}
\hat{A}_{m}(\lambda) & =\hat{A}_{m}(\tilde{\lambda})=F_{m+1}(\tilde{\lambda}) A_{m}(\tilde{\lambda}) F_{m}^{-1}(\tilde{\lambda})= \\
& =F_{m+1}(\tilde{\lambda}) H_{m+1}(\lambda) F_{m+1}^{-1}(\lambda) \hat{A}_{m}(\lambda) F_{m}(\lambda) H_{m}^{-1}(\lambda) F_{m}^{-1}(\tilde{\lambda}),
\end{aligned}
$$

где

$$
H_{m}(\lambda)=\left(\begin{array}{cc}
\frac{\lambda^{2} \hat{b}}{1-\lambda^{2}} & 1 \\
-\hat{a} & \frac{\hat{b}}{1-\lambda^{2}}
\end{array}\right)
$$

Считаем матрицу $F_{m}(\lambda) H_{m}^{-1}(\lambda) F_{m}^{-1}\left(\lambda^{-1}\right)$ скалярной, т.е. имеем

$$
x(\lambda) F_{m}(\lambda)=F_{m}\left(\lambda^{-1}\right) H_{m}(\lambda),
$$

где $x(\lambda)$ - скалярный множитель. Из равенства $x(\lambda) x\left(\lambda^{-1}\right) E=H_{m}(\lambda) H_{m}\left(\lambda^{-1}\right)$ выводим

$$
x(\lambda)=\frac{\lambda \hat{b}}{1-\lambda^{2}} \pm \sqrt{-\hat{a}}=\frac{\eta\left(\lambda^{-1}\right)}{\lambda-\lambda^{-1}} .
$$

Матричное уравнение (3.9) эквивалентно следующей системе уравнений для элементов матрицы $F_{m}(\lambda)$ :

$$
\begin{aligned}
& f_{11}(\lambda)=x^{-1}(\lambda)\left[f_{11}\left(\lambda^{-1}\right) \lambda^{2} \frac{\hat{b}}{1-\lambda^{2}}-\hat{a} f_{12}\left(\lambda^{-1}\right)\right] \\
& f_{12}(\lambda)=x^{-1}(\lambda)\left[f_{12}\left(\lambda^{-1}\right) \frac{\hat{b}}{1-\lambda^{2}}+f_{11}\left(\lambda^{-1}\right)\right] .
\end{aligned}
$$

Пара уравнений на $f_{21}$ и $f_{22}$ аналогична (3.10) при условии замены $f_{11} \rightarrow f_{21}, f_{12} \rightarrow$ $f_{22}$. Система (3.10) решается делением уравнений друг на друга, что дает

$$
\frac{f_{11}(\lambda)}{f_{12}\left(\lambda^{-1}\right)}=\lambda^{-1}, \quad \frac{f_{22}(\lambda)}{f_{22}\left(\lambda^{-1}\right)}=\lambda^{-1} \frac{\hat{b}+\left(\lambda-\lambda^{-1}\right) \sqrt{-\hat{a}}}{\hat{b}-\left(\lambda-\lambda^{-1}\right) \sqrt{-\hat{a}}}
$$

и искомые частные решения вида $f_{12}(\lambda)=\lambda^{-1 / 2}, f_{22}(\lambda)=\lambda^{-1 / 2} \eta$.

СледствиЕ. Матрица $\hat{A}_{m}$ имеет вид

$$
\hat{A}_{m}(\mu)=-\frac{1}{2 \sqrt{-2 \hat{a}}}\left(\begin{array}{cc}
-\mu \sqrt{-2 \hat{a}}+2 \hat{a} v_{m, 1}+\hat{b} & 1 \\
4 \hat{a}-\hat{b}^{2}-2 \hat{a} \mu^{2} & -\mu \sqrt{-2 \hat{a}}-2 \hat{a} v_{m, 1}-\hat{b}
\end{array}\right),
$$

где $\mu=-\left(\lambda+\lambda^{-1}\right) / \sqrt{2}$. 
Таким образом, представлено уравнение

$$
Y_{m+1}(\mu)=\hat{A}_{m}(\mu) Y_{m}(\mu)
$$

решения которого удовлетворяют условию существования точечной симметрии (3.4). В солитонном случае спектр линейной задачи состоит из конечного набора собственных значений, причем этот набор инвариантен относительно обоих потоков по $m$ и по $n$. Спектр, отвечающий решениям уравнения (3.12), не меняется при заменах $\lambda \rightarrow \lambda^{-1}$ или $z \rightarrow-z^{-1}$. Калибровочное преобразование, связывающее $A_{m}$ и $\bar{A}_{m}$, не меняет спектра. Таким образом, приходим к следующему утверждению.

УТВЕРЖДЕНИЕ. Набор точек дискретного спектра, соответствующего граничной задаче (1.1), (3.1), удовлетворяет условию инвариантности относительно инволютивного преобразования $\tilde{\lambda}=\lambda^{-1}$ или, в терминах переменной $z, \tilde{z}=-z^{-1}$.

\section{4. ПОСТРОЕНИЕ СОЛИТОННЫХ РЕШЕНИЙ ГРАНИЧНОЙ ЗАДАЧИ}

Воспользуемся сформулированным выше утверждением и будем строить солитонные решения задачи (1.1), (3.1) с помощью следующей рекуррентной процедуры.

1. Начнем с тривиального решения задачи (1.1), (3.1).

2. Имея на $k$-м шагу решение задачи (1.1), (3.1), определим $(k+1)$-е решение по формуле (2.17) путем добавления к дискретному спектру пары точек $\left\{z_{k+1},-z_{k+1}^{-1}\right\}$, а также зададим дополнительную пару параметров

$$
\beta_{2 k+1}=\frac{b_{2 k+1}}{a^{\prime}\left(z_{k+1}\right)}, \quad \beta_{2 k+2}=\frac{b_{2 k+2}}{a^{\prime}\left(-z_{k+1}^{-1}\right)}
$$

для (2.17).

Таким образом, необходимо ответить на вопрос: каковы условия для величин $b_{2 k+1}, b_{2 k+2}, a^{\prime}\left(z_{k+1}\right), a^{\prime}\left(-z_{k+1}^{-1}\right)$, позволяющие на $k$-м шагу рекурсии получить решение задачи (1.1), (3.1)? Относительно $a^{\prime}\left(z_{k+1}\right), a^{\prime}\left(-z_{k+1}^{-1}\right)$ ответ простой: в солитонном случае, когда спектр исключительно дискретный, $b(z) \equiv 0$, функция $a(z)=W\left(\phi_{n}, \psi_{n}\right) /\left(z^{-1}-z\right)$, где $W$ - вронскиан, аналитична внутри единичного круга, удовлетворяет условию $|a(z)|=1$ при $|z|=1$ и, следовательно, определяется своими нулями:

$$
a(z)=\prod_{j=1}^{k} \frac{z^{2}+z\left(z_{j}^{-1}-z_{j}\right)-1}{z^{2}-z\left(z_{j}^{-1}-z_{j}\right)-1} .
$$

Связь между параметрами $b_{2 k+1}, b_{2 k+2}$ задается следующей леммой (cp. с работой [7]).

ЛЕмма 2. Имеет место соотношение

$$
b_{2 k+2}=-\frac{g_{21}^{(k)}}{b_{2 k+1} g_{12}^{(k)}},
$$


где $g_{i j}^{(k)}$ - элементы матрицы

$$
\begin{gathered}
G^{(k)}=\left[Y^{(k)}\left(\lambda_{2 k+2}\right)\right]^{-1}\left(\begin{array}{ll}
0 & 1 \\
\hat{a} & 0
\end{array}\right) Y^{(k)}\left(\lambda_{2 k+1}\right), \\
Y^{(k)}(\lambda)=\left(\begin{array}{cc}
\psi_{m, 1}^{(k)}(\lambda) & \phi_{m, 1}^{(k)}(\lambda) \\
-\lambda \psi_{m, 0}^{(k)}(\lambda) & -\lambda \phi_{m, 0}^{(k)}(\lambda)
\end{array}\right),
\end{gathered}
$$

$\psi^{(k)}, \phi^{(k)}$ - собственные функции, заданные условиями (2.6) и соответствующие $k$-солитонному решению.

Доказательство проводится путем вычисления матрицы (4.4) при условии $\psi^{(k)}\left(\lambda_{j}\right)=b_{j} \phi^{(k)}\left(\lambda_{j}\right)$.

Лемма 3. С точностъю до постоянного множителя вид функции $G^{(k)}$ не зависит от номера шага итерации.

ДокАЗАТЕльство. Воспользуемся тем, что соседние члены иерархии $Y^{(k)}, k=$ $0,1,2, \ldots$, связаны условием (3.4) и очевидным равенством (см. (3.8))

$$
H^{-1}\left(\lambda^{-1}\right)\left(\begin{array}{ll}
0 & 1 \\
\hat{a} & 0
\end{array}\right) H(\lambda)=\mathrm{const} \cdot\left(\begin{array}{ll}
0 & 1 \\
\hat{a} & 0
\end{array}\right) .
$$

Следовательно,

$$
G^{(k+1)}=\left[Y^{(k)}\left(\lambda_{2 k+2}\right)\right]^{-1} H^{-1}\left(\lambda_{2 k+2}^{-1}\right)\left(\begin{array}{ll}
0 & 1 \\
\hat{a} & 0
\end{array}\right) H\left(\lambda_{2 k+1}\right) Y^{(k)}\left(\lambda_{2 k+1}\right)=\text { const } \cdot G^{(k)},
$$

что и требовалось доказать.

Для окончательного определения параметров солитона нужно еще удовлетворить условию нормировки при $n \rightarrow \pm \infty$. Оценим решения $\Gamma^{(k)}$ линейной системы $(2.16)$ при $n \rightarrow \pm \infty$ :

$$
\Gamma^{(k)} \sim-\frac{z_{k}^{2 n}}{\beta_{k}} \frac{\operatorname{det} A_{k}}{\operatorname{det} A}, \quad n \rightarrow+\infty, \quad\left|z_{k}\right|<1,
$$

где $(A)_{i j}=1 /\left(z_{i}^{-1}-z_{j}\right), A_{k}$ - матрица, полученная из $A$ заменой $k$-го столбца на столбец $(1,1, \ldots, 1)^{\mathrm{T}}$. Подставляя оценку $(4.5)$ в формулу

$$
v_{m, n}=\hat{c}\left(1-\sum_{k=1}^{N} z_{k}^{-2 n-1} \beta_{k} \Gamma^{(k)}\right),
$$

получим условие на $+\infty$ :

$$
v_{m, n} \sim v^{+}=\hat{c} \sum_{\left|z_{k}\right|<1} z_{k}^{-1} \frac{\operatorname{det} A_{k}}{\operatorname{det} A}
$$

Аналогично на $-\infty$ :

$$
v_{m, n} \sim v^{-}=\hat{c} \sum_{\left|z_{k}\right|<1} z_{k} \frac{\operatorname{det} A_{k}}{\operatorname{det} A}
$$


Подставив (4.6) и (4.7) в граничное условие (3.1), получим дополнительное условие для спектра:

$$
\frac{v^{+}}{v^{-}}=\frac{-\hat{b}+\sqrt{\hat{b}^{2}+4 \hat{a}}}{-\hat{b}-\sqrt{\hat{b}^{2}+4 \hat{a}}}=\frac{\sum_{\left|z_{k}\right|<1} z_{k} \operatorname{det} A_{k}}{\sum_{\left|z_{k}\right|<1} z_{k}^{-1} \operatorname{det} A_{k}} .
$$

Теперь имеем следующую теорему.

Теорема. Солитонные решения задачи (1.1), (3.1) порождаются рекуррентной прочедурой (4.1) при следующих условиях на параметры:

1) на каждом шаге $\kappa$ дискретному спектру добавляется пара точек $\left\{z_{k+1},-z_{k+1}^{-1}\right\}$

2) коэффициент $а(z)$ вычисляется по формуле

$$
a(z)=\prod_{j=1}^{N} \frac{z^{2}+z\left(z_{j}^{-1}-z_{j}\right)-1}{z^{2}-z\left(z_{j}^{-1}-z_{j}\right)-1}
$$

3) параметры $b_{k}$ и $b_{k+1}$ связаны равенством $b_{k+1}=-g_{21}^{(k)} / b_{k} g_{12}^{(k)}$ (cм. матрицу $G^{(k)}(4.4)$, вычисляемую ниже отдельно для четных (5.2) и нечетных (5.6) N);

4) выполнено условие нормировки

$$
\frac{\sum_{\left|z_{k}\right|<1} z_{k} \operatorname{det} A_{k}}{\sum_{\left|z_{k}\right|<1} z_{k}^{-1} \operatorname{det} A_{k}}=\frac{-\hat{b}+\sqrt{\hat{b}^{2}+4 \hat{a}}}{-\hat{b}-\sqrt{\hat{b}^{2}+4 \hat{a}}} .
$$

Таким образом, начиная с тривиальных 0- и 1-солитонных решений граничной задачи (1.1), (3.1), мы можем построить две отдельные иерархии решений с четньми и нечетными номерами.

\section{5. КОНКРЕТНЫЕ ФОРМУЛЫ ДЛЯ РЕШЕНИЙ}

Построим набор солитонных решений задачи (1.1), (3.1) с четными номерами. Исходным пунктом является постоянное решение

$$
v^{ \pm}=\frac{-\hat{b} \pm \sqrt{\hat{b}^{2}+4 \hat{a}}}{2 \hat{a}} .
$$

Ему соответствует тривиальная пара собственных функций: $\psi_{n}(z)=z^{n}, \phi_{n}(z)=$ $z^{-n}$. Подставляя их в $(4.3),(4.4)$, получим связь для параметров $b_{k}, b_{k+1}$ :

$$
b_{k+1}=b_{k}^{-1} \text {. }
$$

Так, для 2-солитонного решения имеем формулу

$$
v_{m, n}=\hat{c} \frac{\gamma_{m, n+1}}{\gamma_{m, n}}
$$

где

$$
\begin{aligned}
\gamma_{m, n}=1 & +\beta_{1} \frac{z_{1}^{2 n}}{z_{1}-z_{1}^{-1}}+\beta_{2} \frac{z_{2}^{2 n}}{z_{2}-z_{2}^{-1}}+ \\
& +\beta_{1} \beta_{2}\left(z_{1} z_{2}\right)^{-2 n} \frac{z_{1} z_{2}^{-1}-2+z_{1}^{-1} z_{2}}{\left(z_{1}-z_{1}^{-1}\right)\left(z_{2}-z_{2}^{-1}\right)\left(z_{1}-z_{2}^{-1}\right)\left(z_{2}-z_{1}^{-1}\right)}
\end{aligned}
$$




$$
\begin{gathered}
\hat{c}^{-1}=\hat{c} \hat{a}+\hat{b}, \quad \beta_{j}=\frac{b_{j}}{a^{\prime}\left(z_{j}\right)}, \quad z_{2}=-z_{1}^{-1}, \quad a(z)=\frac{z^{2}+z\left(z_{1}^{-1}-z_{1}\right)-1}{z^{2}-z\left(z_{1}^{-1}-z_{1}\right)-1}, \\
b_{2}=b_{1}^{-1}, \quad z_{1,2}^{2}=\frac{-\hat{b} \mp \sqrt{\hat{b}^{2}+4 \hat{a}}}{-\hat{b} \pm \sqrt{\hat{b}^{2}+4 \hat{a}}} .
\end{gathered}
$$

Теперь опишем рекуррентную процедуру для нечетных номеров. Исходным пунктом будут 1-солитонное решение вида (2.20)

$$
v_{m, n}=\hat{c} \frac{1+\beta_{1} \xi_{1}^{m} z_{1}^{-2 n}}{1+\beta_{1} \xi_{1}^{m} z_{1}^{-2 n+2}},
$$

где

$$
\hat{c}=\frac{-\hat{b} \pm \sqrt{\hat{b}^{2}+4 \hat{a}}}{2 \hat{a}}, \quad z_{1}^{2}=-\hat{a} \hat{c}^{2}, \quad \xi_{1}=\frac{z_{1}+\lambda_{1}}{z_{1}^{-1}-\lambda_{1}^{-1}},
$$

и соответствующая пара собственных функций

$$
\begin{aligned}
\psi_{n} & =-\frac{z^{n}}{\xi_{1}}\left[\alpha \lambda-\frac{p_{m, n-1}}{p_{m-1, n-1}}(z+\lambda)\right], \\
\phi_{n} & =z^{-n}\left[\alpha \lambda^{-1}-\frac{p_{m, n-1}}{p_{m-1, n-1}}\left(\lambda^{-1}-z^{-1}\right)\right],
\end{aligned}
$$

где

$$
p_{m, n}=1+\beta_{1} \xi_{1}^{m} z_{1}^{-2 n}, \quad \alpha=\frac{z_{1}-z_{1}^{-1}}{z_{1} \xi_{1}^{-1}-z_{1}^{-1}}, \quad \lambda^{-1}-\lambda=z+z^{-1} .
$$

Подставив (5.5) в (4.3), (4.4), получим связь для параметров $b_{k}, b_{k+1}$ :

$$
b_{k+1}=\left(\frac{z_{k}^{-1}-\lambda_{k}^{-1}}{z_{k}+\lambda_{k}}\right)^{2} b_{k}^{-1}
$$

что заканчивает описание рекуррентной процедуры сформулированной выше теоремы для нечетных номеров солитонных решений.

Благодарности. Автор выражает признательность И. Т. Хабибуллину за полезные советы и обсуждения. Работа частично поддержана Программой РАН "Математические методы в нелинейной динамике", Фондом содействия отечественной науке и РФФИ (грант № 04-01-00190).

\section{Список литературы}

[1] E. Date, M. Jimbo, T. Miwa, J. Phys. Soc. Japan, 51 (1982), 4116-4124; 4125-4131; 52 (1983), 761-765; 766-771.

[2] Ю.Б. Сурис, Алгебра и анализ, 2:2 (1990), 141-157.

[3] Т. Г. Казакова, ТМФ, 138:3 (2004), 422-436.

[4] Т.Г. Казакова Конечномерные редукиии дискретных систем, Дисс. ... канд. физ.матем. наук, ИМ с ВЦ УНЦ РАН, Уфа, 2005.

[5] I. T. Habibullin, T. G. Kazakova, J. Phys. A, 34 (2001), 10369-10376.

[6] В. Е. Захаров, С. В. Манаков, С.П. Новиков, П.П. Питаевский, Теория солитонов. Метод обратной задачи, Наука, М., 1980.

[7] И. Т. Хабибуллин, ТМФ, 114:1 (1998), 115-125. 